\title{
Flexural waves on a pinned semi-infinite thin elastic plate
}

\author{
D. V. Evans and R. Porter \\ Department of Mathematics, University of Bristol, Bristol, BS8 1TW, UK.
}

\begin{abstract}
The interaction of flexural waves with the edge of a thin semi-infinite elastic plate which is pinned at points along its edge is considered. In particular, it is shown how energy can be fed into edge waves when a plane incident wave is diffracted by points along the edge. Finally, we demonstrate the existence of a new type of Rayleigh-Bloch edge wave for a plate periodically pinned along its edge.
\end{abstract}

Key words: Elastic plates, edge waves, pinned points, Green's functions.

\section{Introduction}

It is well-known that a thin semi-infinite elastic plate in vacuo is capable of supporting an edge wave propagating without change of form along its free edge and decaying exponentially in a direction away from the edge. See, for example, [1] for an early reference. The wavenumber $l$ of this edge wave satisfies $k<l$ where $k$ is the wavenumber of free waves in the plate (in the body of this paper we write $l=k t_{e}$, with $t_{e}>1$ ). It follows that the edge wave cannot be excited by an incident plane wave from infinity which would have a component wavenumber along the edge which is less than $k$. The simplest mechanism for generating the edge wave is some local disturbance such as the prescribed vibration of a given point. To construct this solution we need a Green's function for the semi-infinite half-plane. The Green's function for an infinite thin elastic plate is well-known and is given, for example, by [2]. Less well-known is the Green's function for an elastic semi-infinite plate with a free edge which has been derived by Gunda [3]. A property of both Green's functions is that they are bounded at the source point because of the high order of the differential operator which they satisfy. This property enables problems involving given displacements or forcing of certain points, or the scattering of an incident wave in the presence of one or more pinned points, to be solved in terms of linear combinations of Green's functions at the points in question with or without the incident field.

Email address: d.v.evans@bris.ac.uk, richard.porter@bris.ac.uk (D. V. Evans and R. Porter ). 
In this paper we shall sketch out the derivation of the semi-infinite Green's function and use it to show how either the prescribed forcing of a given point, or the force required to hold a point pinned in the presence of incident waves, gives rise to outgoing cylindrical waves as well as edge waves. By considering the scattering of incident waves by a finite periodic arrangement of points held fixed along the edge, we show how it is possible, for certain combinations of frequencies and point spacings, to increase the amplitude of edge waves which propagate along the remainder of the free edge.

The scattering of incident waves by a periodic infinite array of pinned points along the edge is considered and used to derive a new Rayleigh-Bloch edge wave travelling along the edge and decaying outwards having a wavenumber lying between $k$ and the free edge wavenumber, $l$, determined by [1].

The simplicity of using a single Green's function to represent the application of a force, prescribed or otherwise, at a point on the plate allows us to derive simple expressions for quantities of interest. For example, the condition for the existence of Rayleigh-Bloch edge waves is in expressed mathematically as a real infinite series summing to zero.

\section{Reflection of plane waves by a free edge and the existence of edge waves}

In this section we introduce the governing equations and set up some notation and useful ideas for later sections by reviewing two basic problems namely the reflection of plane waves from, and the existence of edge waves along, the straight edge of an elastic plate.

A thin elastic plate plate occupies the half plane $-\infty<x<\infty, y>0$ with its free edge along $y=0$. There are no external forces acting on the plate. As is usual, we factorise a harmonic time dependence of angular frequency $\omega$ from the out-of-place displacement $U$ by writing $U(x, y, t)=\Re\left\{u(x, y) \mathrm{e}^{-\mathrm{i} \omega t}\right\}$. The complex-valued time-independent displacement $u(x, y)$ satisfies the Kirchhoff-Love equation

$$
\left(\Delta^{2}-k^{4}\right) u(x, y)=0, \quad-\infty<x<\infty, y>0
$$

where $\Delta$ is the two-dimensional Laplacian, $k^{4}=m \omega^{2} / D$ with $m=\rho h$ the mass per unit area of the plate and $D=\frac{1}{12} E h^{3} /\left(1-\nu^{2}\right)$ its bending stiffness in terms of the plate density and thickness, $\rho$ and $h$, and the Young's modulus and Poisson ratio $E$ and $\nu$. It is convenient to rescale lengths on the wavenumber $k$, so that the non-dimensional version of (1) reads

$$
\left(\Delta^{2}-1\right) u(x, y)=0, \quad-\infty<x<\infty, y>0 .
$$

Along the free edge, the vanishing of bending moment and shearing stress requires that

$$
\begin{gathered}
\mathcal{B}(u) \equiv u_{y y}+\nu u_{x x}=0, \quad \text { on } y=0 \\
\mathcal{S}(u) \equiv u_{y y y}+(2-\nu) u_{y x x}=0, \quad \text { on } y=0 .
\end{gathered}
$$

For a plane wave incident from infinity which makes an angle $\psi$ with the positive $x$-axis and is reflected from the edge, the solution is straightforward and is given by

$$
u(x, y)=u_{i n c}(x, y)+u_{s}(x, y)
$$

where the incident wave is described by

$$
u_{i n c}=\mathrm{e}^{-\mathrm{i} y \sin \psi} \mathrm{e}^{\mathrm{i} x \cos \psi} \equiv \mathrm{e}^{\gamma\left(t_{s}\right) y} \mathrm{e}^{\mathrm{i} t_{s} x}
$$

whilst the reflected wave is

$$
u_{s}=\left(R \mathrm{e}^{\mathrm{i} y \sin \psi}+B \mathrm{e}^{-y\left(1+\cos ^{2} \psi\right)^{1 / 2}}\right) \mathrm{e}^{\mathrm{i} x \cos \psi} \equiv\left(R \mathrm{e}^{-\gamma\left(t_{s}\right) y}+B \mathrm{e}^{-\lambda\left(t_{s}\right) y}\right) \mathrm{e}^{\mathrm{i} t_{s} x}
$$


where we have written $t_{s}=\cos \psi$ and introduced the notation

$$
\lambda(t)=\sqrt{1+t^{2}}, \quad \gamma(t)= \begin{cases}-i \sqrt{1-t^{2}}, & |t| \leq 1 \\ \sqrt{t^{2}-1}, & |t|>1 .\end{cases}
$$

Satisfaction of the two edge conditions (3) and (4) provides complicated but explicit expressions for the coefficients $R$ and $B$ in terms of $\psi$. Thus the reflection coefficient can be expressed as

$$
R=-E^{*}\left(t_{s}\right) / E\left(t_{s}\right)
$$

with the asterisk denoting complex conjugation (since $\left|t_{s}\right|<1$ ) so that $|R|=1$, as expected. The amplitude of a wave decaying exponentially away from the edge is found to be

$$
B=2 \gamma\left(t_{s}\right) L_{+}\left(t_{s}\right) L_{-}\left(t_{s}\right) / E\left(t_{s}\right)
$$

where

$$
E(t)=\lambda(t) L_{-}^{2}(t)-\gamma(t) L_{+}^{2}(t), \quad \text { with } \quad L_{ \pm}(t)=(1-\nu) t^{2} \pm 1 .
$$

In the absence of an incident wave, it is possible to construct a solution for a wave travelling along the edge of the semi-infinite plate and decaying exponentially away from it. This is the socalled Konenkov edge wave solution (after [1]) and is well-documented elsewhere in the literature - see for example [4].

In order to construct such a solution consider we replace $t_{s}$, associated with the scattering angle $\psi$ with a value of $t_{e}>1$ in the general solution (5) and discard the incident wave. Then the general form for the displacement of the edge wave is given by

$$
u_{e}^{ \pm}(x, y)=\left(C \mathrm{e}^{-\gamma\left(t_{e}\right) y}+D \mathrm{e}^{-\lambda\left(t_{e}\right) y}\right) \mathrm{e}^{ \pm \mathrm{i} t_{e} x} .
$$

in terms of coefficients $C$ and $D$, to be determined. It now follows after some algebra that the conditions (3) and (4) are satisfied if and only if $E\left(t_{e}\right)=0$ where $E(t)$ is real for $t>1$. The value of $t_{e}$ can be determined explicitly as the real positive root of

$$
t_{e}^{4}=\frac{1-3 \nu+2 \sqrt{2 \nu^{2}-2 \nu+1}}{(1-\nu)^{2}(3+\nu)}
$$

and is a function of $\nu$ only. It is interesting to note that if $\nu=\frac{1}{3}-$ a reasonable value for Poisson's ratio - that $t_{e}=(81 / 80)^{1 / 8}=1.0015 \ldots$ More generally, $t_{e} \sim 1+\frac{1}{16} \nu^{4}+O\left(\nu^{5}\right)$ and so $t_{e}$ is always only just above unity. Since $t_{e}>1$ its associated (dimensional) wave speed $c_{e}=\omega / k t_{e}<c=\omega / k$, the free wave speed in the elastic plate. For this reason it is called a subsonic or slow wave.

The edge wave is a solution to a homogeneous boundary-value problem, and so the coefficients $C$ and $D$ are not determined uniquely. For the purposes of this paper, we make a particular choice for $C$, and hence $D$, and refer to this as being an edge wave of unit amplitude. We write

$$
u_{e}^{ \pm}(x, y)=F\left(t_{e}, y\right) \mathrm{e}^{ \pm \mathrm{i} t_{e} x}
$$

where

$$
F(t, y)=\frac{1}{2}\left(L_{+}(t) \mathrm{e}^{-\gamma(t) y}-L_{-}(t) \mathrm{e}^{-\lambda(t) y}\right) .
$$

such that $F(t, 0)=1$ for all $t$. The symbols \pm refer to edge waves propagating ${ }^{1}$ in the positive and negative $x$-directions (respectively) along the edge.

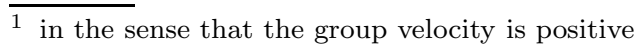




\section{Scattering of incident waves by pinned points}

Clearly, an edge wave cannot be excited by a plane incident wave $\left(t_{e}>1\right.$ whilst $\left.t_{s}<1\right)$. However, if one or more points of the plate are pinned in the presence of an incident wave, or given a prescribed time-harmonic displacement in the absence of an incident wave, the periodicity of $u$ defined by (5) in the $x$-direction is destroyed and there is no reason why energy cannot be fed into edge wave solutions proportional to $u_{e}^{ \pm}$defined in (14).

\section{The Green's function for a semi-infinite elastic plate}

To consider such problems in which a point (or points) on a semi-infinite elastic plate is given a prescribed force, either to hold the point pinned or to provide it with a required displacement, we need an expression for the Green's function for a semi-infinite plate satisfying the free edge conditions. Such a Green's function has been derived previously by [3] using an elegant method which merits a brief description here. First, we consider the Green's function $g_{\infty}(x, y ; \xi, \eta)$ describing a point forcing at the point $(\xi, \eta)$ on a plate of infinite extent, satisfying

$$
\left(\Delta^{2}-1\right) g_{\infty}(x, y ; \xi, \eta)=\delta(x-\xi) \delta(y-\eta)
$$

and describing outward travelling waves at large distances. This is well known and given by

$$
g_{\infty}(x, y ; \xi, \eta)=\frac{1}{8} \mathrm{i}\left(H_{0}^{(1)}(r)-H_{0}^{(1)}(\mathrm{i} r)\right),
$$

where $H_{0}^{(1)}(\cdot)$ is the Hankel function of order zero, and $r=\left((x-\xi)^{2}+(y-\eta)^{2}\right)^{1 / 2}$. A useful alternative to (17) is the integral representation

$$
g_{\infty}(x, y ; \xi, \eta)=\frac{1}{8 \pi} \int_{-\infty}^{\infty} \mathrm{e}^{\mathrm{i}(x-\xi) t}\left(\frac{\mathrm{e}^{-\gamma(t)|y-\eta|}}{\gamma(t)}-\frac{\mathrm{e}^{-\lambda(t)|y-\eta|}}{\lambda(t)}\right) \mathrm{d} t
$$

where $\gamma$ and $\lambda$ are given by (8). It follows, from the asymptotic properties of the Hankel function for small and large arguments that

$$
g_{\infty}(x, y ; \xi, \eta) \sim \frac{1}{8} \mathrm{i}+O\left(r^{2} \ln r\right), \quad \text { as } r \rightarrow 0
$$

which is bounded at the origin, whilst

$$
g_{\infty}(x, y ; \xi, \eta) \sim \frac{1}{8} \mathrm{i}\left(\frac{2}{\pi r}\right)^{1 / 2} \mathrm{e}^{i(r-\pi / 4)}, \quad \text { as } r \rightarrow \infty .
$$

The desired Green's function, $g(x, y ; \xi, \eta)$ for the semi-infinite plate must satisfy the edge conditions (3) and (4) in addition to the governing equation (16) in $y, \eta>0$. We write

$$
g=g_{o d d}+w, \quad \text { where } \quad g_{o d d}=g_{\infty}(x, y ; \xi, \eta)-g_{\infty}(x, y ; \xi,-\eta)
$$

and clearly $g_{\text {odd }}=\mathcal{B}\left(g_{\text {odd }}\right)=0$ on $y=0$. It follows that $g$ will satisfy all the required conditions provided $w$ is regular in $y>0$ and satisfies

$$
\left.\begin{array}{l}
\mathcal{B}(w)=0, \quad \text { and } \\
\mathcal{S}(w)=-\mathcal{S}\left(g_{\text {odd }}\right) \equiv-\frac{1}{2 \pi} \int_{-\infty}^{\infty} \mathrm{e}^{\mathrm{i}(x-\xi) t} F(t, \eta) \mathrm{d} t
\end{array}\right\} \quad \text { on } y=0,-\infty<x<\infty
$$

with $F(t, y)$ defined by (15). By choosing

$$
w(x, y ; \xi, \eta)=-\frac{1}{\pi} \int_{-\infty}^{\infty} \mathrm{e}^{\mathrm{i}(x-\xi) t}\left(A(t) \mathrm{e}^{-\lambda(t)|y-\eta|}+B(t) \mathrm{e}^{-\gamma(t)|y-\eta|}\right) \mathrm{d} t
$$




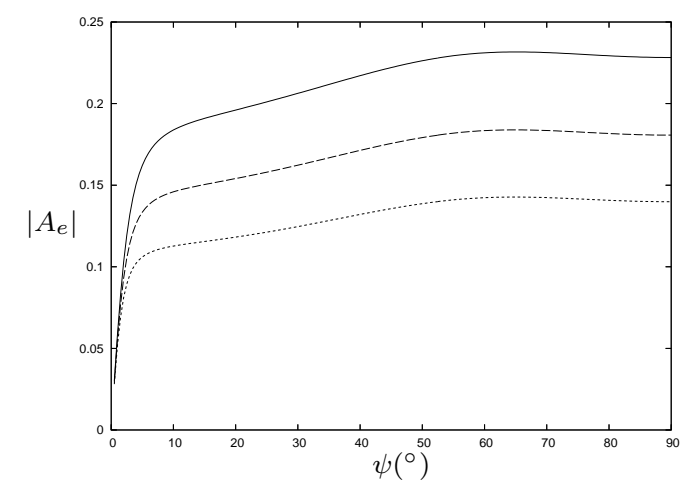

Fig. 1. Edge wave amplitude coefficient $\left|A_{e}\right|$ against incident wave angle $\psi$ (in degrees) for a pinned origin and with $\nu=0.33$ (upper curve), 0.3 (centre) and 0.27 (lower).

where $A(t)$ and $B(t)$ are determined from the conditions (22) it is found, after some algebra, that

$$
w(x, y ; \xi, \eta)=-\frac{1}{\pi} \int_{-\infty}^{\infty} \mathrm{e}^{\mathrm{i}(x-\xi) t} \frac{F(t, \eta) F(t, y)}{E(t)} \mathrm{d} t .
$$

The path of integration passes above the pole at $t=-t_{e}$ and below the pole at $t=t_{e}$ to ensure the radiation condition (that edge waves carry energy towards infinity) is met. Like $g_{\infty}$, the Green's function $g$ is bounded at $(\xi, \eta)$ and can be used to solve a variety of problems involving pinned or forced points.

Scattering by a pinned point on the edge

In the simplest example we assume the origin is pinned. Then $g$ reduces to

$$
g(x, y ; 0,0) \equiv \hat{g}(x, y)=-\frac{1}{\pi} \int_{-\infty}^{\infty} \mathrm{e}^{\mathrm{i} x t} \frac{F(t, y)}{E(t)} \mathrm{d} t .
$$

The displacement of the plate when a plane wave is incident from infinity making an angle $\psi$ with the positive $x$-axis on an edge pinned at the origin is written as

$$
u(x, y)=u_{i n c}(x, y)+u_{s}(x, y)+A_{0} \hat{g}(x, y)
$$

where $u_{i n c}$ and $u_{s}$ are given by (6) and (7) with $t_{s}=\cos \psi$ as before. The free edge conditions are already satisfied by $u(x, y)$ and it is simply the imposition of the condition $u(0,0)=0$ that determines the constant $A_{0}$ (which may be regarded as the force required to hold the point fixed) as

$$
A_{0}=-(1+R+B) / \hat{g}(0,0)
$$

where $R$ and $B$ are given by (10) and (11) whilst

$$
\hat{g}(0,0)=-\frac{2}{\pi} \int_{0}^{\infty} \frac{1}{E(t)}
$$

the contour being indented below the pole at $t=t_{e}$. The integral is convergent, since

$$
E(t) \sim-|t| t^{2}(1-\nu)(1+3 \nu), \quad \text { as }|t| \rightarrow \infty .
$$

The amplitude of the edge waves generated from the pinned origin is evident from letting $|x| \rightarrow \infty$ with $y$ bounded in (25) where the dominant contribution comes from poles at $t= \pm t_{e}$. It follows that

$$
u(x, y) \sim A_{e} u_{e}^{ \pm}(x, y), \quad x \rightarrow \pm \infty, \quad \text { where } A_{e}=-\frac{2 \mathrm{i} A_{0}}{E^{\prime}\left(t_{e}\right)}
$$




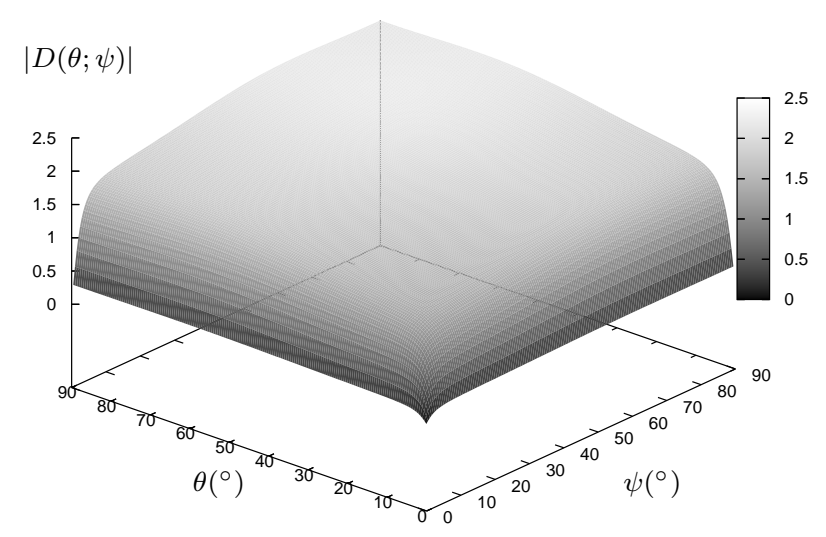

Fig. 2. Plot of the diffraction coefficient $|D(\theta ; \psi)|$ for a pinned point at the origin.

is the edge wave amplitude coefficient, proportional to $A_{0}$ defined by (27). A plot of $\left|A_{e}\right|$ against $\psi$ is given in figure 1 for a range of values of Poisson's ratio, showing the largest amplitudes occurring for $\psi$ close to normal incidence.

Away from the edge, we may determine the diffraction coefficient being a measure of the amplitude of the outgoing circular waves generated by the pinned origin. Again, attention focuses on (26), but we now work in the limit of $r \rightarrow \infty, 0<\theta<\pi$, where $x=r \cos \theta$ and $y=r \sin \theta$. A standard application of the steepest descent method gives

$$
u(x, y) \sim D(\theta ; \psi)\left(\frac{\pi}{2 r}\right)^{1 / 2} \mathrm{e}^{\mathrm{i}(r-\pi / 4)}, \quad r \rightarrow \infty, 0<\theta<\pi
$$

where

$$
D(\theta ; \psi)=-\frac{A_{0} \gamma\left(t_{o}\right) L_{+}\left(t_{o}\right)}{2 E\left(t_{o}\right)}, \quad t_{o}=\cos \theta
$$

defines the diffraction coefficient (sometimes called the directivity) which measures the amplitude factor for the observation angle $\theta$ in terms of the incident angle $\psi$. The dependence on $\psi$ is incorporated in $A_{0}$, defined by (27) which can also be written as

$$
A_{0}=\frac{4 \gamma\left(t_{s}\right) L_{+}\left(t_{s}\right)}{E\left(t_{s}\right) \hat{g}(0,0)}
$$

Hence, it can be seen that the diffraction coefficient satisfies the reciprocity relation, $D(\theta ; \psi)=$ $D(\psi ; \theta)$, a result which can be established independently with the use of Green's theorem. The symmetric diffraction coefficient $D(\theta ; \psi)$ is plotted in figure 2 for $\nu=\frac{1}{3}$.

Clearly, the asymptotic form given in (31) does not hold in the limits $\theta \rightarrow 0$ or $\pi$ as the nature of the outgoing waves changes from circular waves with algebraic decay with distance from the origin to edge waves with no decay along the edge. Mathematically, this manifests itself in the steepest descent method with the poles at $t= \pm t_{e}$ close to the points \pm 1 having an increasingly significant effect as the saddle point in the integral at $t=t_{o}$ tends to \pm 1 . Formally, an extra corrective term needs to be added to (31) which accounts for the influence of the poles associated with the edge waves. For a recent detailed description of this procedure, see [5]. For example, as $\theta \rightarrow 0$ (or $t_{o} \rightarrow 1$ ), the correction to the far-field (31) is given by

$$
u_{c}(r, \theta)=\frac{1}{2} A_{e} \mathrm{e}^{\mathrm{i} r}\left\{W\left(\sqrt{2 r} \mathrm{e}^{\mathrm{i} \pi / 4} \sin \frac{1}{2}\left(\theta-\mathrm{i} v_{e}\right)\right)-\mathrm{e}^{\mathrm{i} \pi / 4} \csc \frac{1}{2}\left(\theta-\mathrm{i} v_{e}\right) / \sqrt{2 \pi r}\right\}
$$

where $t_{e}=\cosh v_{e}$ defines $v_{e}>0, A_{e}$ is the edge wave amplitude defined in $(30)$ and $W(z)=$ $\mathrm{e}^{-z^{2}} \operatorname{erfc}(-\mathrm{i} z)$ is a scaled complementary error function - see [6]. Note that $u_{c}$ itself is a solution 


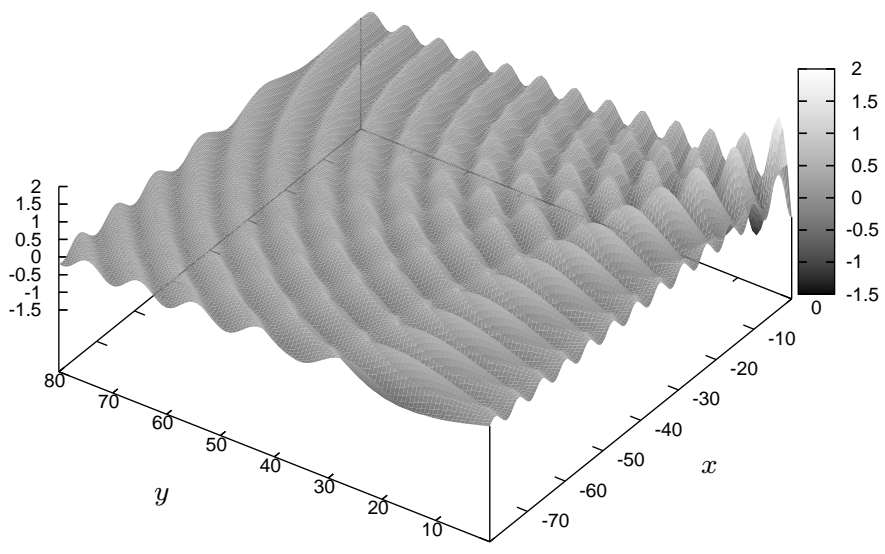

Fig. 3. Plot of the wave field due to the effect of the pinned origin for a wave incident normal to the edge $y=0$.

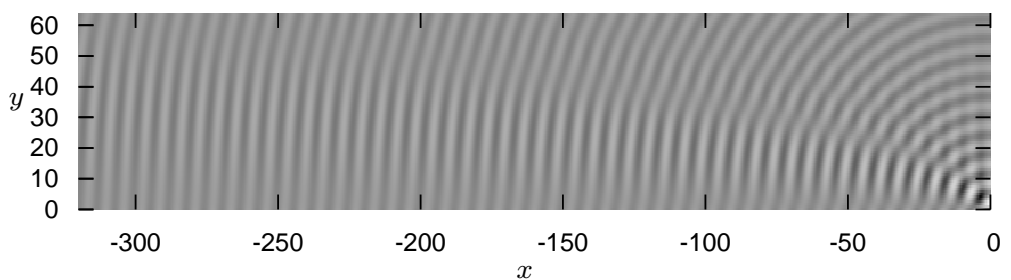

Fig. 4. Plot of the wave field along the edge $y=0$ for normal incidence.

of the Helmholtz equation and therefore the plate equation. For $\theta \rightarrow \pi$, simply replace $\theta$ by $\pi-\theta$ in (34).

Using $W(z) \sim \mathrm{i} /(z \sqrt{\pi})$ for $|z| \rightarrow \infty$ when $-\frac{1}{4} \pi<\arg (z)<\frac{5}{4} \pi$, it can be easily verified that the leading order contribution of $u_{c}$ for $0<\theta<\pi$ is zero. That is to say, that for any $\theta$ not equal to 0 or $\pi$, there is no contribution from $u_{c}$ to the diffraction coefficient. However, in the limit $\theta \rightarrow 0$ or $\pi$, the relation $W(z)=2 \mathrm{e}^{-z^{2}}-W(-z)$ can be used to show that the leading order contributions from $u_{c}$ are just the edge wave solutions $A_{e} u_{e}^{ \pm}(x, y)$. Thus, the correction $u_{c}$ to (31) provides a uniform description of the far-field displacement for all values of $\theta$ between zero and $\pi$.

As an example of the wave field created by the pinned point, we plot in figure 3 the real part of the complex surface displacement due to the final term in (26) for a wave normally incident on the edge $\left(\psi=\frac{1}{2} \pi\right)$. This snapshot in time of the wave field is symmetrical in $x$ and drawn only for values of $x \leq 0$. The wavelength of free waves is $2 \pi$, whilst the wavelength of the edge waves excited by the point source at the origin is fractionally larger at $2 \pi t_{e}$. The effect of the superposition of edge waves which decay with a slow exponent away from $y=0$ and the circular diffracted waves from the origin manifests itself in the interference patterns in figure 3. A more panoramic view of the wave field along this edge is given in figure 4 in which the persistence of a wave travelling along the edge, with slow decay away from the edge, can be seen. 

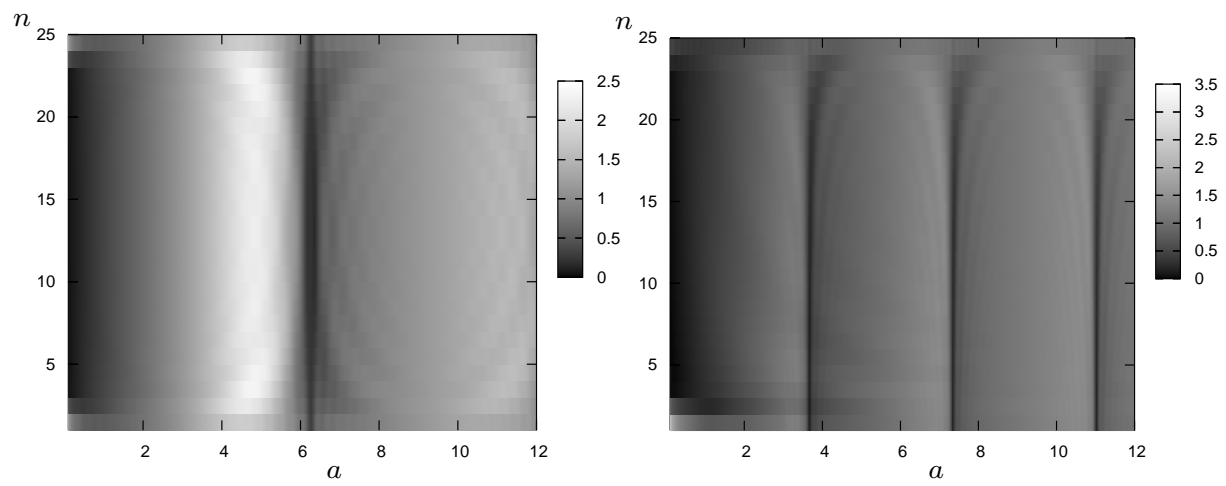

Fig. 5. Intensity plot of $\left|A_{n}\right|$ with pin number $n$ and against non-dimensional spacing, $a$, with $N=25$ pinned points: (a) $\psi=90^{\circ}$; (b) $\psi=45^{\circ}$.

Scattering by multiple points

It is, of course, a straightforward matter to generalise the above analysis to consider scattering by multiple pinned points. For example, if the edge is pinned at $N$ equally-spaced points $(n a, 0)$ for $n=1, \ldots, N$ (remembering the dimensional spacing is $a / k$ ) we write

$$
u(x, y)=u_{i n c}(x, y)+u_{s}(x, y)+\sum_{n=1}^{N} A_{n} g(x, y ; n a, 0)
$$

then the coefficients $A_{n}$ are given as the solution to the system of equations

$$
\sum_{n=1}^{N} A_{n} \hat{g}((m-n) a, 0)=-\mathrm{e}^{\mathrm{i} t_{s} m a}(1+R+B), \quad m=1, \ldots, N .
$$

An extension of the methods used for a single pin can be used here to calculate properties of interest. For example, the amplitude of edge waves generated by the arrangement of $N$ equallyspaced points towards $\pm \infty$ is given by

$$
A_{e}^{ \pm}=-\frac{2 \mathrm{i}}{E^{\prime}\left(t_{e}\right)} \sum_{n=1}^{N} A_{n} \mathrm{e}^{\mp \mathrm{i} t_{e} n a} .
$$

Results showing the size of the coefficients $\left|A_{n}\right|$ (normalised against the value of $A_{0}$ in (27) for a single pinned point), for 25 pinned points, against $a$ for two incident wave angles of $\psi=90^{\circ}$ and $\psi=45^{\circ}$ are presented in figure 5. Key features to note are that for any given value of $a$ and $\psi$, the values of $\left|A_{n}\right|$ do not vary much across most of the array, a fact we will exploit in a moment. Secondly, there are values of $a$, depending upon $\psi$ where the values of $A_{n}$ become relatively small, denoted by the dark vertical stripes in figure 5 .

In figures 6 and 7 , we show the variation of $\left|A_{e}^{ \pm}\right|$with $a$, being the amplitude of edge waves generated by $N$ points and computed using (37). In figure 6 , the waves are normally incident on the pins $\left(\psi=90^{\circ}\right)$ and the values of $\left|A_{e}^{+}\right|$and $\left|A_{e}^{-}\right|$are identical. The three curves are for $N=25,50$ and 100 pinned points which appear to oscillate around a common path over nearly all values of $a$, apart from at $a=2 \pi$, where the height of the peaks increase with increasing values of $N$. In figure 7, a fixed number, $N=25$, of points is chosen but now $\psi$ is varied. The values of $\left|A_{e}^{+}\right|$and $\left|A_{e}^{-}\right|$now differ, whilst the characteristic peaks occur at different values of $a$.

The value of $a$ at which peaks occur can be predicted using some basic reasoning. We assume that the coefficients $A_{n}$ have approximately the same magnitude for all $n$, as suggested by figures 5. Indeed if the periodic array were infinite in extent, then this would be the case, and it would 


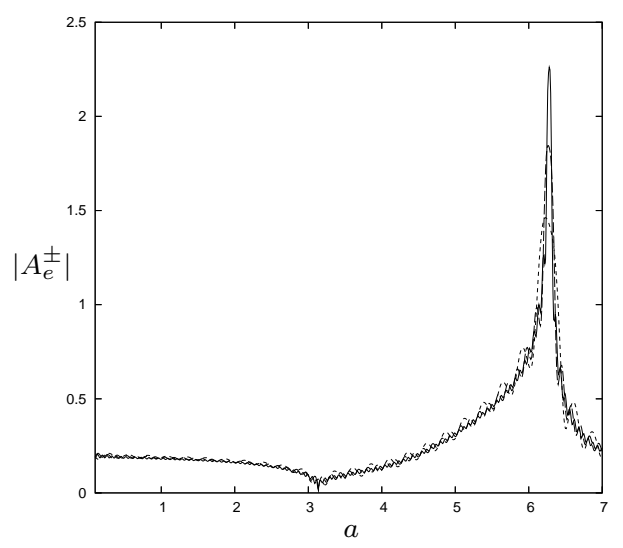

Fig. 6. Variation of $\left|A_{e}^{ \pm}\right|$with $a$ for $\psi=90^{\circ}$ with $N=100$ (solid), $N=50$ (long dash), $N=25$ (short dash).

also follow that the only difference in the values of $A_{n+1}$ and $A_{n}$ would be the phase difference, $\mathrm{e}^{\mathrm{i} t_{s} a}$ of the incident wave. Thus, we assume for the large finite array that $A_{n} \approx \bar{A} \mathrm{e}^{\mathrm{i} t_{s} n a}$ in which case

$$
A_{e}^{ \pm} \approx-\frac{2 \mathrm{i} \bar{A}}{E^{\prime}\left(t_{e}\right)} \sum_{n=1}^{N} \mathrm{e}^{\mathrm{i}\left(t_{s} \mp \mathrm{i} t_{e}\right) n a}=-\frac{2 \mathrm{i} \bar{A}}{E^{\prime}\left(t_{e}\right)}\left(\frac{\mathrm{e}^{\mathrm{i} \beta^{ \pm} N}-1}{1-\mathrm{e}^{-\mathrm{i} \beta^{ \pm}}}\right)
$$

where $\beta^{ \pm}=\left(t_{s} \mp t_{e}\right) a$. Thus, $\left|A_{e}^{ \pm}\right|$takes its maximum values when $\beta^{ \pm}=2 p \pi, p \in \mathbb{Z}$ and this translates to the condition $a=2 p \pi /\left(t_{e} \mp \cos \psi\right)$ for the maximum values of $\left|A_{e}^{ \pm}\right|$, with the bracketed term in (38) evaluating to $N$. The values of $a$ predicted by this formula tally exactly with those values of $a$ in figures 7 at which the peaks occur, although the height of the peaks (e.g. figure 6) do not scale linearly in $N$ - evidently there is some dependence of $\bar{A}$ on $N$.

Other features in figures 6 and 7 can be explained from (38). For example, the curves oscillate with with a period in $a$ given by $2 \pi /\left(N\left(t_{e} \mp \cos \psi\right)\right)$, which increases with $N$. The dark vertical stripes in figure 5 correspond to 'resonance' in figures 6 and 7 . At these values of $a$, the force required to pin each point in the array is relatively small on account of the superposition of edge waves generated by each point being in phase with one another. In figure 8 we show the function $u-u_{s}-u_{i n c}$ which represents a snapshot in time of the deflection on the elastic plate due to the $N=25$ pinned points on the edge. The non-dimensional spacing between points is $a=3.66$, chosen to correspond to resonance in this case where $\psi=45^{\circ}$. This diffracted field shows a generally complicated pattern. Note the contrast between relatively large amplitude edge waves along the edge $y=0$ for $x<0$ travelling to $x=-\infty$, and those with small amplitudes for $x>88$ (i.e. beyond the far right of the array of pinned points).

We can easily estimate the effect of giving just one of the periodically pinnned points, say $(p a, 0), p \in\{1, \ldots, N\}$, a unit displacement in the absence of the incident wave. Thus the displacement can be written

where $B_{n}^{p}$ satisfies

$$
u^{p}(x, y)=\sum_{n=1}^{N} B_{n}^{p} g(x, y ; n a, 0)
$$

$$
\sum_{n=1}^{N} B_{n}^{p} \hat{g}((m-n) a, 0)=\delta_{m p}, \quad m=1 \ldots N .
$$

Then it easily follows that

$$
A_{n}=-(1+R+B) \sum_{p=1}^{N} B_{n}^{p} \mathrm{e}^{\mathrm{i} t_{s} p a} .
$$



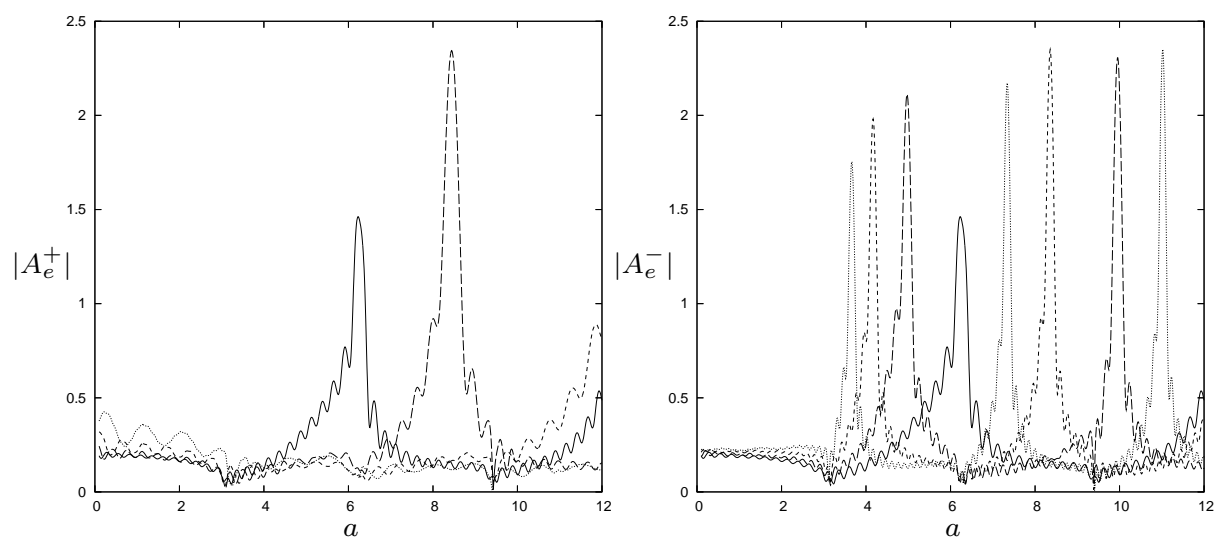

Fig. 7. Variation of $\left|A_{e}^{ \pm}\right|$with $a$ for $N=25$ points with $\psi=90^{\circ}$ (solid), $\psi=75^{\circ}$ (long dash), $\psi=60^{\circ}$ (short dash), $\psi=45^{\circ}$ (dotted).

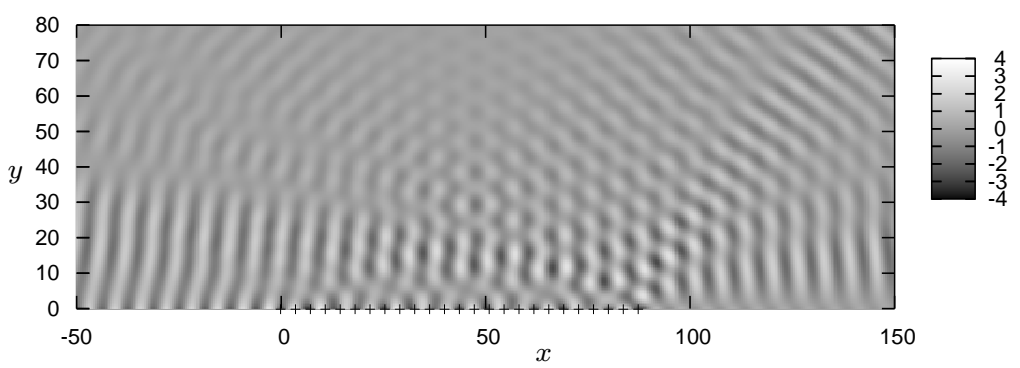

Fig. 8. Snapshot of plate deflection generated by the pinned points only $\left(u-u_{s}-u_{\text {inc }}\right)$ in the case of $N=25$ pinned points (shown as crosses) along the edge $y=0$ at $x=(n-1) a, n=1, \ldots, N$ for $a=3.66$ and $\psi=45^{\circ}$.

Thus the scattering of an incident wave by $N$ pins can be expressed in terms of the solution of the unit displacement of each of the pins in turn when the remainder are fixed.

For an infinite periodic array of pinned points, located at $(n a, 0), n \in \mathbb{Z}$ it follows from (35) that

$$
u(x, y)=u_{i n c}(x, y)+u_{s}(x, y)+\sum_{n=-\infty}^{\infty} A_{n} g(x, y ; n a, 0) .
$$

In this case, the quasi-periodicity in $x$ allows one to argue that there is only a phase factor, equal to that inherited from the component of the incident wave in $x$, across any one period, and hence it must follow that

$$
A_{n}=A_{0} \mathrm{e}^{\mathrm{i} t_{s} n a} .
$$

Application of $u(m a, 0)=0$, for $m \in \mathbb{Z}$ to now gives

$$
-\frac{2 A_{0}}{a} S\left(t_{s}, a\right)=-(1+R+B) \equiv 4 \gamma\left(t_{s}\right) L_{+}\left(t_{s}\right) / E\left(t_{s}\right)
$$

as an equation determining the value of $A_{0}$, where

$$
S(t, a)=-\frac{a}{2} \sum_{n=-\infty}^{\infty} \mathrm{e}^{-\mathrm{i} t n a} \hat{g}(n a, 0)
$$

and

$$
\hat{g}(n a, 0)=-\frac{1}{\pi} \int_{-\infty}^{\infty} \frac{\mathrm{e}^{\mathrm{i} a n u}}{E(u)} \mathrm{d} u
$$


follows from (25). Use of the Poisson summation formula,

$$
\sum_{n=-\infty}^{\infty} \int_{-\infty}^{\infty} \mathrm{e}^{\mathrm{i} n u} f(u) \mathrm{d} u=2 \pi \sum_{n=-\infty}^{\infty} f(2 n \pi),
$$

allows us to evaluate the slowly-convergent series in (42) to give

$$
S(t, a)=\sum_{n=-\infty}^{\infty} \frac{1}{E\left(t_{n}\right)}, \quad \text { with } t_{n}=t+2 n \pi / a .
$$

The summation in (44) is convergent, on account of the behaviour of $E(t)$ for large argument, as noted in (29).

It is convenient to define scattering angles, $\psi_{n}$, for $n \in \mathcal{N}$, the subset of $\mathbb{Z}$ for which $\left|t_{n}\right|<1$ by $\cos \psi_{n}=t_{n}$. When, as in equation (41), $t=t_{s}=\cos \psi$, the set $\mathcal{N}$ is non-empty for all values of $a$ since, by definition $t_{0}=t_{s}$ with $\left|t_{0}\right|<1$. As the non-dimensional spacing $a$ increases the number of values of $t_{n}$ such that $\left|t_{n}\right|<1$ increases and so the set $\mathcal{N}$ grows. Increasing $a$ is equivalent, in dimensional terms, to fixing the spacing and increasing the wavenumber, $k$, or vice versa.

To see the effect on the total scattered field we note from (39) that this is

$$
\begin{aligned}
u(x, y)-u_{i n c}(x, y) & =u_{s}(x, y)+A_{0} \sum_{n=-\infty}^{\infty} \mathrm{e}^{\mathrm{i} t_{s} n a} g(x, y ; n a, 0) \\
& =R \mathrm{e}^{\mathrm{i} t_{s} x} \mathrm{e}^{-\lambda\left(t_{s}\right) y}+B \mathrm{e}^{\mathrm{i} t_{s} x} \mathrm{e}^{-\gamma\left(t_{s}\right) y}-\frac{2 A_{0}}{a} \sum_{n=-\infty}^{\infty} \frac{F\left(t_{n}, y\right)}{E\left(t_{n}\right)} \mathrm{e}^{\mathrm{i} t_{n} x} \\
& \sim R \mathrm{e}^{\mathrm{i}(x \cos \psi+y \sin \psi)}-\frac{A_{0}}{a} \sum_{n \in \mathcal{N}} \frac{L_{+}\left(t_{n}\right)}{E\left(t_{n}\right)} \mathrm{e}^{\mathrm{i}\left(x \cos \psi_{n}+y \sin \psi_{n}\right)}
\end{aligned}
$$

as $y \rightarrow \infty$, where Poisson's formula has been used again and exponentially decaying terms have been neglected, whilst $A_{0}$ is determined from (41). Thus, for large $y$ the scattered field only involves plane waves arising from $n \in \mathcal{N}$ and the periodic array of pinned points acts as a classical diffraction grating would. The reflection coefficient corresponding to the scattering angle $\psi_{n}$ is $R_{n}=R \delta_{n 0}-A_{0} L_{+}\left(t_{n}\right) / E\left(t_{n}\right) a$. For small $a$ (corresponding in dimensional terms to small spacing or small $k$ ) there is just one scattered plane wave corresponding to $n=0$.

Conservation of energy requires that the condition

$$
\sum_{n \in \mathcal{N}} \frac{\gamma\left(t_{n}\right)}{\gamma\left(t_{0}\right)}\left|R_{n}\right|^{2}=1
$$

be met. This result can be shown to hold exactly from the formulation presented here. For example, in the simplest case when there is only one mode propagating, use of the definitions for $R$ and $A_{0}$ immediately give $R_{0}=\left[-E^{*}\left(t_{s}\right) E\left(t_{s}\right) S\left(t_{s}, a\right)+2 \gamma\left(t_{s}\right) L_{+}\left(t_{s}\right)\right] / E^{2}\left(t_{s}\right) S\left(t_{s}, a\right)$ which can be rearranged to give $R_{0}=-\left(1+E^{*}\left(t_{s}\right) S^{\prime}\right) /\left(1+E\left(t_{s}\right) S^{\prime}\right)$ where $S^{\prime}=S\left(t_{s}, a\right)-1 / E\left(t_{s}\right)$ is real. It follows that $\left|R_{0}\right|=1$. We do not present a proof of the general result (46) since it is significantly more complicated and requires lots of tedious algebra.

\section{Edge waves along a periodically pinned edge}

In a manner similar to the way in which the edge wave solution was developed for an elastic plate with a free edge, we can consider the possibility of edge waves travelling along a periodically pinned edge. Thus, we write 


$$
u(x, y)=A_{0} \sum_{n=-\infty}^{\infty} \mathrm{e}^{-\mathrm{i} t_{p} n a} g(x, y ; n a, 0)
$$

neglecting the components $u_{i n c}$ and $u_{s}$ in the scattering problem, but invoking the quasiperiodicity in the coefficients $A_{n}$, the periodicity now associated with $t_{p}$, a value to be determined, rather than $t_{s}$ which was previously defined in terms of the scattering angle $\psi$. Application of the pinned condition $u(m a, 0)=0$ for $m \in \mathbb{Z}$ leads to the condition

$$
S\left(t_{p}, a\right)=0
$$

where $S$ is defined by (44). It is satisfaction of this condition which determines the value of $t_{p}$, a task made easier if we place the restriction we $1<t_{p}<2 \pi / a-1$. Then it follows that $\left|t_{n}\right|>1\left(t_{n}\right.$ is defined by (44)) and hence $E\left(t_{n}\right)$ is real for all $n \in \mathbb{Z}$. Hence $S\left(t_{p}, a\right)$ is real and the possibility of meeting the condition (48) is significantly enhanced. The requirement $t_{p}<2 \pi / a-1$ implies the restriction $a<\pi$ which, in dimensional terms, means the product of the spacing and wavenumber $k$ be less than $\pi$.

There are good reasons to suppose that (48) can be satisfied for values of $t_{p}$ lying in the interval $\left(1, t_{e}\right)$ where $t_{e}$ is associated with the Konenkov edge wave along a free edge, defined by (13). These come about from a detailed analysis of the components $1 / E\left(t_{n}\right), n \in \mathbb{Z}$, of the sum $S(t, a)$. Thus, we note that for $|t| \geq 1,1 / E(t)$ is an even function, with $\mathrm{d} / \mathrm{d} t(1 / E(t))>0$ for $t \geq 1$, $t \neq t_{e}$. Also, $E(1)=\sqrt{2} \nu^{2}$ whilst $E\left(t_{e}\right)=0$ and we note the large argument asymptotics of $E(t)$ expressed by (29). Then, provided $1<t_{p}<t_{e}$ and $t_{p}<2 \pi / a-t_{e}$, there is just one positive contribution to the sum $S\left(t_{p}, a\right)$ from the term $1 / E\left(t_{0}\right)$ (of course, $t_{0} \equiv t_{p}$ ) the rest being all negative. The values $1 / E\left(t_{0}\right)$ can take, as $t_{p}$ varies from 1 to $t_{e}$, range from $\left(\sqrt{2} \nu^{2}\right)^{-1}$ to infinity. Thus, it can be argued that an appropriate choice of $t_{p}$ in $\left(1, t_{e}\right)$ can balance the remaining negative contributions of $1 / E\left(t_{n}\right), n \neq 1$ to $S\left(t_{p}, a\right)$ to give a net result of zero. However, all but $1 / E\left(t_{-1}\right)$ of the remaining terms in the series contribute a relatively small amount to $S\left(t_{p}, a\right)$. A crude estimate, using the asymptotic form (29) for all but two of the terms in the series, gives

$$
\begin{aligned}
& S\left(t_{p}, a\right) \approx \frac{1}{E\left(t_{p}\right)}+\frac{1}{E\left(t_{p}-2 \pi / a\right)} \\
& \quad-\frac{1}{(1-\nu)(1+3 \nu)}\left\{\sum_{n=1}^{\infty} \frac{1}{(1+2 n \pi / a)^{3}}+\sum_{n=2}^{\infty} \frac{1}{(2 n \pi / a-1)^{3}}\right\} .
\end{aligned}
$$

For realistic values of $\nu$, the negative contribution from the second line above comes no where near overhauling the much larger positive contribution from the first term and so any chance of satisfying $S=0$ relies on the second term being sufficiently negative. This requires $t_{p}-2 \pi / a$ to be less than, but close to the value $-t_{e}$, and this then requires $a$ to be close to but just less than $2 \pi /\left(1+t_{e}\right)$ which itself is less than but close to $\pi$, on account of $t_{e}$ being only just greater than unity. Furthermore, if we consider the limit $t_{p} \rightarrow t_{e}$ from below, the balance of the first and second increasingly large terms in (49) requires $t_{p}-2 \pi / a \rightarrow-t_{e}$ from below and so $a \rightarrow \pi / t_{e}$ from below.

The analysis above is backed up by the numerical results in which solutions of (48) are found to exist in only a small range of values, bounded below by $a_{l}$, given by $S\left(1, a_{l}\right)=0$. For example, with $\nu=\frac{1}{3}$, we find that $a_{l}=3.13216$, whilst the maximum value $a$ takes is given by $\pi / t_{e}$ with $t_{e}=1.00155 \ldots$ for this particular value of $\nu$.

In figure 9 we show dispersion curves for the edge waves that exist along the periodically pinned edge. The curves show the computed values of $t=t_{p}$ as a function of non-dimensional spacing, $a$. The axes are arranged in this particular way to identify with the usual presentation of results in terms of dimensional quantities (for example [7]), in which the vertical axis represents $k a$ and the horiztonal axis is $\beta a$ (that is, $t$ represents $\beta / k$ ), a now being the physical spacing, $k$ the free 


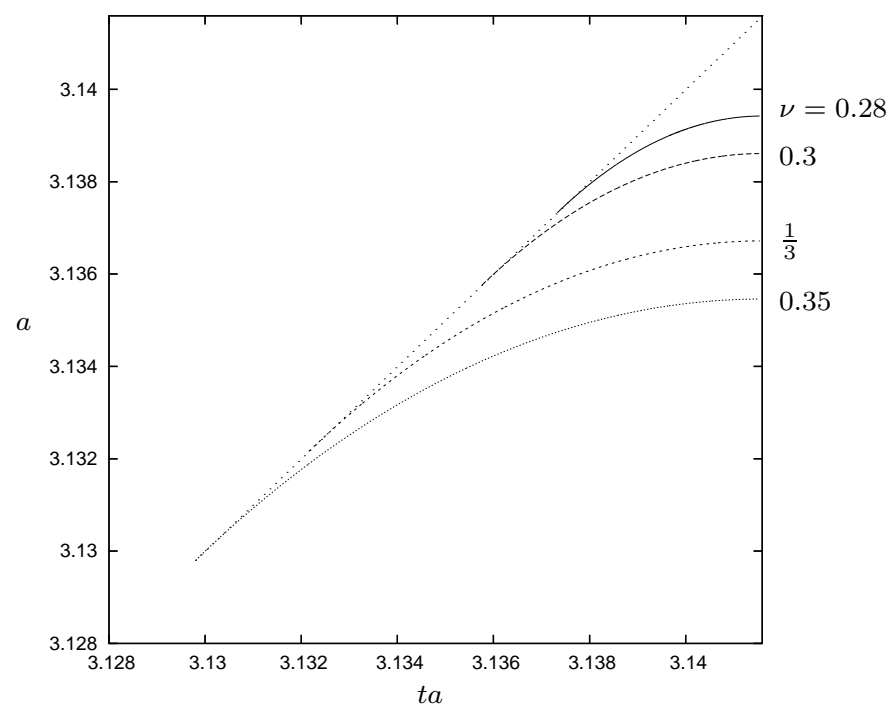

Fig. 9. Dispersion curves, $t=t_{p}$ against dimensionless spacing $a$, for edge waves along a periodically pinned edge of a semi-infinite plate for various values of $\nu$. The dotted line is the cut-off, $t=1$.

space wavenumber and $\beta$ the 'Rayleigh-Bloch' wavenumber. This latter terminology arises since this particular type of edge wave, which exists along a periodic array, are usually referred to in the literature as Rayleigh-Bloch waves.

The form of the Rayleigh-Bloch edge waves can be determined from (47), where $A_{0}$ is arbitrary. Use of Poisson's summation formula gives

$$
u(x, y)=-\frac{2 A_{0}}{a} \sum_{n=-\infty}^{\infty} \frac{F\left(t_{n}, y\right)}{E\left(t_{n}\right)} \mathrm{e}^{\mathrm{i} t_{n} x}
$$

with $t_{n}=t_{p}+2 \pi n / a$. The displacement is exponentially decaying in $y$, since $\left|t_{n}\right|>1$ by construction, with the two terms $n=0,1$ dominating the series. Indeed, in the limit as $t_{p} \rightarrow t_{e}$ $\left(\right.$ as $\left.a \rightarrow \pi / t_{e}\right)$

$$
u(x, y) \rightarrow 2 F\left(t_{e}, y\right) \sin \left(t_{e} x\right) \equiv u_{e}^{+}(x, y)-u_{e}^{-}(x, y)
$$

where $A_{0} \rightarrow 0$ in such a way as to scale out the effect of the denominator tending to zero. That is to say, the Rayleigh-Bloch wave in this case is just a standing wave comprised of the difference of two Konenkov edge waves travelling in opposing direction whose nodal lines, $x=n a$, are perpendicular to the edge and connect at the edge with the pinned points. The fact that $A_{0} \rightarrow 0$ is no surprising, as no force is required to hold the nodal points of a standing wave.

The standing wave solution in (51) also applies to points pinned at $x=2 n a$, for example (more generally, for $x=$ pna with $p=1,2,3, \ldots$ ), in which every other nodal point on the edge is pinned. This solution requires $a=2 \pi / t_{e}$, which is beyond the range of values for $a$ argued earlier in order that $S\left(t_{p}, a\right)$ be real. However, the situation can be resolved mathematically by constructing a standing wave solution from the outset, odd about $x=0$, whilst insisting that $t_{p}=0$, in which case we replace $(47)$ with

$$
u(x, y)=A_{0} \sum_{n \neq 0}\{g(x, y ; n a, 0)-g(-x, y ; n a, 0)\}
$$

which leads to the condition (48) with

$$
S(0, a)=\sum_{n \neq 0} \frac{1}{E\left(t_{n}\right)}, \quad t_{n}=2 \pi n / a,
$$


which is clearly real when $a<2 \pi$. The condition $S(0, a)=0$ is only satisfied by $a=2 \pi / t_{e}$ in which case (52) reduces to (51), as required.

In other physical settings involving periodic arrays [8], [9], it has been shown that the presence of Rayleigh-Bloch waves can cause near-resonance in the corresponding scattering problem involving large finite periodic arrays. We have been unable to produce any such results here, although this may be due to the very limited range of values of $a$ for which Rayleigh-Bloch solutions may be obtained (figure 9).

\section{Conclusions}

It has been shown that the Green's function for a semi-infinite thin elastic plate can be used to investigate a variety of interesting problems involving pinned and forced points. In particular, we have shown that plane waves incident on a pinned point on the straight edge of an elastic plate can generate edge waves which radiate energy to infinity along the edge. We have also demonstrated the existence of a new type of edge wave along a periodically pinned edge.

Besides those problems investigated here, problems involving semi-infinite periodic arrays can be treated using methods such as those described in [11], where interest now lies in how much incident plane wave energy can be fed into Rayleigh-Bloch edge waves.

It should also be possible to use distributions of the Green's function and various combinations of derivatives of Green's function to investigate continuously pinned lines and narrows cracks with free edges in the proximity of a free edge, although such problems are non-trivial (see [10] for example).

\section{Acknowledgements}

The authors thank Dr. Ian Thompson of Loughborough University for providing them with useful notes on the uniform asymptotics needed for determining the far field scattering in section 3.

\section{References}

[1] Yu. K. Konenkov, A Rayleigh-type flexural wave. Sov. Phys. Acoust. 6 (1960) 122-123.

[2] A.N. Norris \& C. Vermula, Scattering of flexural waves on thin plates. J. Sound Vib. 181, 115-125 (1995).

[3] R. Gunda, S.M. Vijayaker, R. Singh and J.E. Farstad, "Harmonic Green's function of a semi-infinite plate with clamped or free edges" J. Acoust. Soc. Am. 103(2), 888-899 (1998).

[4] A.N. Norris, V.V. Krylov and I.D. Abrahams, "Flexural edge waves and Comments on 'A new bending wave solution for the classical plate equation'" J. Acoust. Soc. Am. 107(3), 1781-1784 (2000).

[5] I. Thompson, "An improved uniform approximation for diffraction integrals" Proc. Roy. Soc. Lond. A 462, 1341-1353 (2006).

[6] M.A. Abramowitz and I. Stegun, Handbook of mathematical functions, Dover (1964).

[7] D.V. Evans and M. Fernyhough, "Edge waves along periodic coastlines. Part 2" J. Fluid. Mech. 297, 301-325 (1995).

[8] D.V. Evans and R. Porter, "Penetration of flexural waves through a periodically constrained thin elastic plate in vacuo and floating on water" J. Eng. Math. 58, 317-337 (2005).

[9] I. Thompson, C.M. Linton and R. Porter, "A new approximation method for scattering by long arrays" (submitted).

[10] R. Porter and D.V. Evans, "Diffraction of flexural waves by finite straight cracks in an elastic sheet over water" J. Fluids and Structures 23(2), 309-327 (2007).

[11] C.M. Linton and P. A. Martin, "Semi-infinite arrays of isotropic point scatterers" SIAM J. Appl. Math. 64(3), 1035-1056 (2004). 